\title{
Random Key Cuckoo Search for the Quadratic Assignment Problem
}

\author{
Aziz Ouaarab*", Bela"id Ahiod*, Xin-She Yang ${ }^{\dagger}$ \\ *LRIT, Associated Unit to the CNRST(URAC) no 29, Mohammed V-Agdal University, Morocco \\ ${ }^{+}$School of Science and Technology, Middlesex University, The burroughs, London NW4 4BT, UK \\ ${ }^{\text {IS}}$ School of Technology Essaouira, Cadi Ayyad University. Morocco \\ aziz.ouaarab@gmail.com; Ahiod@fsr.ac.ma; x.yang@mdx.ac.uk
}

\begin{abstract}
This paper proposes an adaptation of the Random- Key Cuckoo Search (RKCS) algorithm for solving the famous Quadratic Assignment Problem (QAP). We used a simplified and efficient random-key encoding scheme to convert a continous space (real numbers) into a combinatorial space. We also considered the displacement of a solution in both spaces by using Le'vy flights. The performance of the RKCS for QAP is tested against a set of benchmarks of QAP from the well-known QAPLIB library, and the comparison with a set of other methaheuristics is also carried out.

Index Terms-Nature-Inspired Metaheuristic, Cuckoo Search, Le'vy Flights, Combinatorial Optimization, Quadratic Assign- ment Problem, Random-Key.

\section{Introduction}

NP-hard problems [17] are very challenging to solve. It is also the most complicated among combinatorial optimization problems. The main difficult of such problems is that the number of combinations grows exponentially with the problem size. Quadratic assignment problem [7] is one of the problems that belongs to this class.

Quadratic Assignment Problem (QAP) is a combinatorial optimization problem that is applied to solve various problems in many fields such as Steinberg Wiring Problem [5], Hospital Layout [13], Dartboard Design [12], and many other applica- tions [7], [14]. Problems such as QAP do not have an efficient algorithm to solve them exactly. It is practically very difficult to get a solution of optimal quality and in a reduced runtime simultaneously. This requires some heuristic algorithms that can find good (not necessarily optimal) solutions in a good runtime by trial and error. Approximate algorithms such as metaheuristics [4] are actually the best choice to solve many combinatorial optimization problems. They are characterized by their simplicity and flexibility while demonstrating remark- able effectiveness. Many metaheuristics are proposed to solve QAP. Existing studies include Genetic Algorithms (GA) [1] that used a sequential constructive crossover, a modified Particle Swarm Optimisation (PSO) [21], Ant Colony Optimisation (ACO) [11], and many other examples [22], [15].
\end{abstract}

Among the most difficult issues that arises when solving a combinatorial optimization problem with a metaheuristic, is how to move in the combinatorial solution space without affecting the performance of 
the metaheuristic. Several meta- heuristics are designed in principle for continuous optimization problems. So, the question is how to treat combinatorial problems properly without losing the good performance of these metaheuristics. In this paper, we used the Random-Key Cuckoo Search (RKCS) algorithm by using the random key encoding scheme to represent a position, found by the cuckoo search algorithm, in the QAP solution space.

This work presents a novel approach using the improved cuckoo search algorithm [26], based on random keys [3], with a simple local search procedure to solve QAP.

The rest of this paper is organized as follows: Section 2 introduces briefly the QAP. Section 3, first, introduces the standard cuckoo search and its improved version. Section 4 presents the random-key encoding scheme, while Section 5 describes how CS solves QAP by using Random key. Then, Section 6 presents results of numerical experiments on a set of QAP benchmarks from the QAPLIB library [8]. Finally, Section 7 concludes with some discussions and future directions.

\section{Quadratic Assignment Problem}

The Quadratic Assignment Problem is a combinatorial optimisation problem, which tries to minimize the total cost of building and operating the facilities knowing that the benefit resulting from an economic activity at any site is depending on the sites of the other facilities. The solution space in QAP is considered as a set of all potential assignments of the facilities to the possible sites.

A selected solution $S$ is the permutation $\varphi$ of a given set $Q=\{1,2, \ldots, N\}$ where $N$ is the instance dimension, it is also the number of sites and facilities, $\varphi(i)=k$ means that the facility $i$ is assigned to the site $\mathrm{k}$.

The objective problem is to find a permutation $\varphi=(\varphi(1), \varphi(2), \ldots, \varphi(N))$ that minimizes

$$
\sum_{i=1}^{N} \sum_{j=1}^{N} a_{i j} b_{\phi(i) \phi(j)}
$$

where $a$ is the flow matrix, and aij is the flow between facilities $i$ and $j$, and b denotes the distance matrix. So, the distance from facility $i$ to $j$ takes the value of $b \varphi(i) \varphi(j)$.

Here, $\varphi(i)$ is the location assigned to facility i. The aim is to minimize the sum of products flow $\times$ distance [30].

\section{Cuckoo Search Algorithm}

In the aim to increase their survival chances and reduce the probability of abandoning eggs by the host birds, cuckoos adopt many strategies and tricks. These strategies are mimicked successfully and designed in the well known Cuckoo Search (CS) algorithm [34]. Cuckoo search introduces Le'vy flights [28] for generating a new good solution. Le'vy flights, named after the French mathematician Paul Le'vy, represent a model of random walks characterized by their step lengths which obey a power-law distribution [6]. CS is widely applied to solve many combinatorial optimization problems such as Travelling Salesman Problem [25], [27], Flow Shop Schedul- ing Problem [20], Knapsack Problem [19].

The first version of CS, which is developed by Xin-She Yang and Suash Deb, is summarized as the following ideal rules: (1) Each cuckoo lays one egg at a time and selects a nest randomly; (2) The best nest with the 
Aziz Ouaarab, Belaid Ahiod, Xin-She Yang, Random Key Cuckoo Search for the Quadratic Assignment Problem. Transactions on Machine Learning and Artificial Intelligence, Vol 5 No 4 August (2017); p: 842-851

highest quality of egg can pass onto the new generations; (3) The number of host nests is fixed, and the egg laid by a cuckoo can be discovered by the host bird with a probability pa $\in[0,1]$. pa is also a switch parameter to control the balanced combination of local

explorative random walk and the global explorative random walk. The local random walk can be written as :

$$
x_{i}^{t+1}=x_{i}^{t}+\alpha s \otimes H\left(p_{a}-\epsilon\right) \otimes\left(x_{j}^{t}-x_{k}^{t}\right),
$$

where $x_{j}{ }^{t}$ and $x_{k}{ }^{t}$ are two different solutions selected randomly by random permutation, $H(u)$ is a Heaviside function, $\epsilon$ is a random number drawn from a uniform distribution, and $s$ is the step size. On the other hand, the global random walk is carried out by using Lévy flights

Where

$$
x_{i}^{t+1}=x_{i}^{t}+\alpha L(s, \lambda) \text {, }
$$

$$
L(s, \lambda)=\frac{\lambda \Gamma(\lambda) \sin (\pi \lambda / 2)}{\pi} \frac{1}{s^{1+\lambda}}, \quad\left(s \gg s_{0}>0\right) .
$$

Here $\alpha>0$ is the step size scaling factor, which should be related to the scales of the problem of interest. Lévy flights have an infinite variance with an infinite mean [34]. Here $s_{0}$ is a constant, which can be take as 0.01 to 0.1 .

Before applying CS to solve QAP, as described in the Algorithm 1, we will consider an improved version of CS [26]. This improvement introduces a new category $p_{c}$ of cuckoos that can engage a kind of surveillance on nests likely to be a host. So, around the pertinent solutions, this portion $p_{c}$ intensify the search process to find a new better solution via Lévy flights.

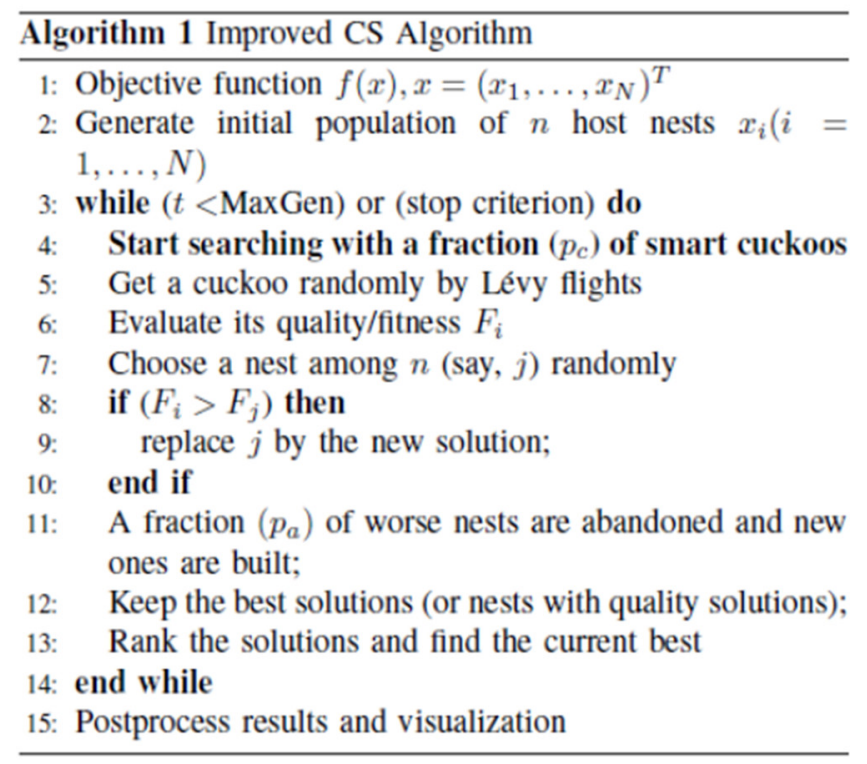




\section{Random-Key Encoding Scheme}

Random-key encoding scheme [3], [27] is an interesting procedure that can be useful to pass from a continuous space to the combinatorial space. In general, the position in the continuous space is represented by a vector of real numbers. To have a projection in the combinatorial space, random-key (RK) associates each real number with a weight. These weights are used to generate one combination as a solution. The random real numbers drawn uniformly from $[0,1)$ compose a vector showed in Figure 1. On the other hand, the combinatorial vector is composed of integers ordered according to the weights of real numbers in the first vector, illustrated in the Figure 1.

\begin{tabular}{|c|c|c|c|c|c|c|}
\hline Random keys: & 0.8 & 0.5 & 0.7 & 0.1 & 0.4 & 0.2 \\
\hline Deco & 6 & 4 & 5 & 1 & 3 & 2 \\
\hline
\end{tabular}

Figure. 1. Random key encoding scheme

\section{Random Key Cs For Qap}

A QAP solution is a vector of $N$ integers. Each integer is the facility index and its order in the vector is the corresponding site index. By considering Figure 1, we can say that resulting vector in this figure is a QAP solution. So to move from the current solution to a new one we can just perturb the first vector that contains the real numbers. This perturbation is performed via Lévy flights. In the case of big jumps we per- form a random pairwise interchanges of vector integers. It is a series of chained swaps $\phi_{\xi(1) \xi(2)}$ , $\phi_{\xi(2) \xi(3)}, \ldots, \phi_{\xi(\rho-1) \xi(\rho)}$, where $\xi(1), \xi(2), \ldots, \xi(\rho)$ is a sequence of random integers between 1 and $\mathrm{N}$ such that $\xi(i)=\xi(j), i, j=1,2, \ldots, \rho, i=j, 1<\rho \leq N$. The mutated solution $\pi$ can thus be represented as a composition $\left(\left(\left(\left(\left(\pi \oplus \phi_{\xi(1) \xi(2)}\right) \oplus \phi_{\xi(2) \xi(3)}\right) \oplus, \ldots\right) \oplus \xi(\mathrm{i}) \xi(\mathrm{i}+1)\right) \oplus \ldots\right) \oplus \phi_{\xi(\rho-1) \xi(\rho)}$, where $\pi$ is the current solution. This series of chained swaps is called the controlled chained mutation (CCM) [23]. The parameter $\rho$ is the mutation rate. This allows an improved way to balance the search for solutions in local areas as well as global areas.

To detect the best solution in the found area, a simple local search (Steepest Descent [2]) is performed. In this local search method we used 'swap' move as described in Figure 2 (In this example we chose to swap the facilities of the sites 2 and 6, which are 1 and 9.) which moves from a placement $\varphi$ to a neighbour placement $\pi$ by applying a swap between facilities $r$ and $s$ :

$$
\begin{aligned}
& \pi(k)=\phi(k), \quad \forall k \neq r, s \\
& \pi(r)=\phi(s), \quad \pi(s)=\phi(r) \\
& \begin{array}{|l|l|l|l|l|l|l|l|l|l|l|l|}
\hline & 1 & 2 & 3 & 4 & 5 & 6 & 7 & 8 & 9 & 10 & 11 \\
\hline 2 & 10 & 1 & 5 & 3 & 8 & 9 & 4 & 0 & 11 & 7 & 6 \\
\hline
\end{array} \\
& \begin{array}{l}
\text { SwapMove }\left(\mathrm{i}_{=2,} \mathrm{j=6}\right) \\
\qquad \begin{array}{|l|c|c|c|c|c|c|c|c|c|c|c|c|}
\hline 2 & 10 & 9 & 5 & 3 & 8 & 1 & 4 & 0 & 11 & 7 & 6 \\
\hline
\end{array}
\end{array}
\end{aligned}
$$

To gain some precious time, estimations are generally performed. They restrict positively the choice of passing to a new good solution. 
In the case of symmetrical matrices with a null diagonal the cost $\delta(\phi, r, s)$ of a move is given by:

$$
\begin{aligned}
\delta(\phi, r, s) & =\sum_{i=1}^{N} \sum_{j=1}^{N}\left(a_{i j} b_{\phi(i) \phi(j)}-a_{i j} b_{\pi(i) \pi(j)}\right) \\
= & 2 \cdot \sum_{k \neq r, s}\left(a_{s k}-a_{r k}\right)\left(b_{\phi(s) \phi(k)}-b_{\phi(r) \phi(k)}\right)(8)
\end{aligned}
$$

In the minimization case, the swap move is done only if the new solution cost is less than the current one. Obviously, this process is repeated until no further improvement is possible or when a given number of steps is reached.

Steepest Descent is a simple local search method that can be easily trapped in a local minimum and, generally, it cannot find good quality solutions. We choose this simplified local search "Steepest Descent" method to show the performance of CS combined with RK for QAP. It allowed us to generate solutions of good quality, without introducing an advanced local search method.

\section{Experimental Results}

We will show some results of running RKCS to solve a set of bechmark instances [29], [31], [32], [10], [9], [13], [18], [33], [24] of QAP from the QAPLIB library [8]. Forty-six instances are considered with sizes ranging from 12 to 100 facilities. The numerical value in the name of an instance represents the number of provided facilities, e.g., the instance named sko90 has 90 facilities. We note that for each instance, 10 independent runs are carried out. These results describe the performance of this first version approach.

In Table II, we compare RKCS with two algorithms based on Genetic Algorithm [1] (SCX) and Ant Colonies [15] (HAS- QAP). Another comparisons are carried out to a Particle Swarm Optimization based algorithm [16] (HPSO) in Ta- ble III. We have implemented RKCS algorithm using Java under 32 bit MS

Windows Seven operating system. Experi- ments are conducted on a laptop with Intel(R) Core ${ }^{\top \mathrm{M}} 2$ Duo $2.00 \mathrm{GHz} \mathrm{CPU}$, and $3 \mathrm{~GB}$ of RAM. SCX algorithm has been encoded in Visual $\mathrm{C}++$ and run on a PC with Intel ${ }^{(\mathrm{R})} \mathrm{Core}^{(\mathrm{TM})}$ i7-3770 CPU @ 3.40GHz and 8.00GB RAM under MS Windows Seven. For HAS-QAP it was not possible to obtain the hardware configurations used in the experiments. HPSO has been run on Intel pentium core 2 Duo Q9950 $(2.83 \mathrm{GHz})$.

TABLE I

PARAMETER SETTINGS FOR RKCS ALGORITHM.

\begin{tabular}{lll}
\hline Parameter & Value & Meaning \\
\hline$n$ & 20 & Population size \\
$p_{c}$ & 0.6 & Portion of smart cuckoos \\
$p_{a}$ & 0.2 & Portion of bad solutions \\
MaxGen & 500 & Maximum number of iterations \\
$\alpha$ & 0.01 & Step size \\
$\lambda$ & 1 & Index \\
$\rho$ & Levy & Mutation rate \\
\hline
\end{tabular}


The properly selected parameter values used for the experiments of RKCS algorithm are shown in Table I. These values are selected, based on some preliminary trials, and gave the best results concerning both the solution quality and the computational time. In each case study, 10 independent runs of RKCS with these parameters are carried out.

TABLE II

COMParing RKCS with GeNetic Algorithm (SCX) [1] AND ANT COLONIES (HAS-QAP) [15].

\begin{tabular}{llllllll}
\hline & \multicolumn{2}{c}{ SCX } & \multicolumn{2}{c}{ HAS-QAP } & \multicolumn{2}{c}{ RKCS } \\
\hline Instance & Bkv & PDbest(\%) & time(s) & PDbest(\%) & time(s) & PDbest(\%) & time(s) \\
\hline tai20a & 703482 & 4.50 & 6 & 1.483 & 3 & $\mathbf{0 . 0}$ & 20 \\
tai20b & 122455319 & 6.56 & 6 & 0.243 & 3 & $\mathbf{0 . 0}$ & 0.5 \\
tai25a & 1167256 & 4.58 & 9 & 2.527 & 5 & $\mathbf{0 . 0}$ & 39 \\
tai25b & 344355646 & 5.24 & 9 & 0.133 & 5 & $\mathbf{0 . 0}$ & 1 \\
tai30a & 1818146 & 4.29 & 13 & 2.600 & 9 & $\mathbf{0 . 0}$ & 74 \\
tai30b & 637117113 & 8.78 & 13 & 0.260 & 9 & $\mathbf{0 . 0}$ & 5 \\
tai35a & 2422002 & 4.95 & 18 & 2.969 & 15 & 0.92 & 96 \\
tai35b & 283315445 & 5.00 & 18 & 0.343 & 15 & $\mathbf{0 . 0}$ & 11 \\
tai40a & 3139370 & 4.53 & 24 & 3.063 & 24 & 0.79 & 141 \\
tai40b & 637250948 & 7.30 & 24 & 0.280 & 24 & $\mathbf{0 . 0}$ & 11 \\
tai50a & 4938796 & 4.51 & 37 & 3.487 & 50 & 1.34 & 258 \\
tai50b & 458821517 & 5.60 & 37 & 0.291 & 50 & $\mathbf{0 . 0}$ & 163 \\
tai60a & 7205962 & 4.54 & 54 & 3.686 & 88 & 1.27 & 433 \\
tai60b & 608215054 & 5.12 & 54 & 0.313 & 90 & $\mathbf{0 . 0}$ & 133 \\
tai80a & 13499184 & 4.35 & 95 & 2.996 & 220 & 1.53 & 1067 \\
tai80b & 818415043 & 6.63 & 95 & 1.108 & 225 & $\mathbf{0 . 0}$ & 1033 \\
sko42 & 15812 & - & - & 0.654 & 25 & $\mathbf{0 . 0}$ & 87.06 \\
sko49 & 23386 & - & - & 0.661 & 45 & 0.05 & 245.17 \\
sko56 & 34458 & - & - & 0.729 & 69 & 0.02 & 356.25 \\
sko64 & 48498 & - & - & 0.504 & 105 & $\mathbf{0 . 0 0}$ & 521.09 \\
sko72 & 66256 & - & - & 0.702 & 153 & 0.11 & 820.53 \\
sko81 & 90998 & - & - & 0.493 & 222 & 0.09 & 1090.11 \\
sk090 & 115534 & - & - & 0.591 & 307 & 0.04 & 1469.63 \\
\hline
\end{tabular}

In the following three tables of experimental results, the first column 'Instance' shows the name of the instance, while the column 'Bkv' shows the optimal solution value taken from the QAPLIB [8]. In Table II, the column 'PDbest(\%)' gives the percentage deviation of the best solution value over the optimal solution value of 10 runs, and the column 'time' denotes the average run time. In Table III, the remaining columns 'mean' and 'Stdev' are the mean and standard deviation of solution cost (expressed as Average Percentage Deviation (APD), which is the percentage by which the cost exceeds the Bkv). The last Table IV which gives statistic results of testing RKCS on some QAP instances contains nine columns. The column 'best' shows the value of the best found solution. The column 'average' gives the average solution value of the 10 independent runs, the column 'worst' shows the value of the worst solution found by RKCS. The column 'SD' denotes the standard deviation. The column 'PDav (\%)' denotes the percentage deviation of the average solution value over the optimal solution value of 10 runs. Bold values indicate that the solutions found have the same length as the Bkv. The percentage deviation of a solution to the best known solution (or optimal solution if known) is given by the Equation 9 .

$$
P D \text { solution }(\%)=\frac{\text { solution value }- \text { best } \text { known solution value }}{\text { best } \text { known solution value }} \times 100
$$

The three tables show that RKCS can be a useful tool to switch from continuous search space to combinatorial one. It also facilitates a better control of the balance between intensification and diversification through local and global random walks. 


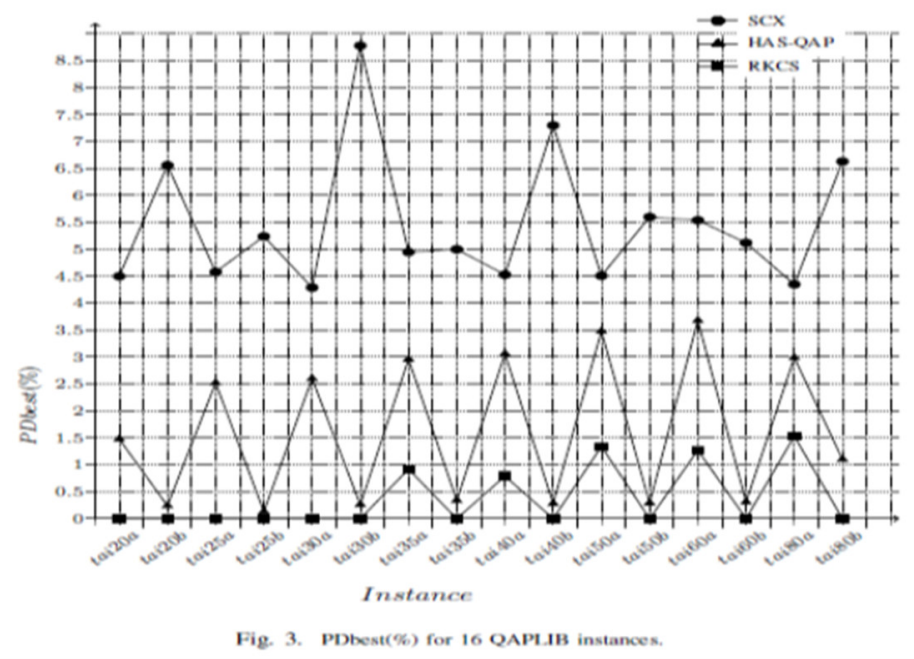

SCX performs a modified sequential constructive crossover operators in genetic algorithms, without using any robust local search technique to improve the solution quality. HAS-QAP is a hybrid ant colony system coupled with a simple local search that consists in applying a complete neighborhood examination twice with first improving strategy [15]. These two methods are compared with RKCS in Table II and Figure 3. We can observe that the difference between RKCS and the two methods is significant. This can be explained basically by a good balance between intensification and diversification, an intelligent use of Lévy flights and the reduced number of parameters.

Table III, shows the comparison of RKCS and HPSO. In this hierarchical particle swarm optimization, particles are arranged in a tree-like hierarchy, where the better-performing particles float towards the top of the hierarchy, and each particle is influenced by its immediate parent in the tree [16]. We have used in this comparison, the mean and the standard deviation. The results prove that RKCS outperforms HPSO in solving the tested QAP instanced. One of the advantages of the RKCS is the relatively independent of cuckoos in the search process for the best solution, and the use of several search strategies adopted by each category of cuckoos, without using any advanced local search method.

These results show that RKCS can be adapted easily to solve QAP. Therefore, we can say that the random-key en- coding scheme can be a very useful tool for switching from continuous to combinatorial spaces. It allows operators of the continuous space to behave freely, then projecting the changes made by these operators in the combinatorial space. It also facilitates a better control in balancing intensification and diversification through Lévy flights, which make intensified small steps in a limited region followed by a big explorative jump to a distant region. Using the real numbers, Lévy flights can easily act with the notion of distance, and can define clearly small or big steps. Then RK projects these changes in the space of QAP solutions. 
TABLE III

COMPARISON OF RKCS WITH HPSO A PARTICLE SWARM OPTIMISATION BASED ALGORITHM [16].

\begin{tabular}{|c|c|c|c|c|c|c|c|c|c|c|c|}
\hline \multirow[b]{2}{*}{ Instance } & \multirow[b]{2}{*}{ Bkv } & \multicolumn{2}{|c|}{ HPSO } & \multicolumn{2}{|c|}{$R K C S$} & \multirow[b]{2}{*}{ Instance } & \multirow[b]{2}{*}{ Bkv } & \multicolumn{2}{|c|}{$H P S O$} & \multicolumn{2}{|c|}{$R K C S$} \\
\hline & & mean & Stdev & mean & Stdev & & & mean & Stdev & mean & Stdev \\
\hline bur26a & 5426670 & 1.9 & 0.22 & 0.0 & 0.0 & tai $12 \mathrm{a}$ & 224416 & 8.11 & 1.42 & 0.0 & 0.0 \\
\hline bur26b & 3817852 & 1.96 & 0.41 & 0.0 & 0.0 & tai $12 b$ & 39464925 & 4.82 & 1.79 & 0.0 & 0.0 \\
\hline bur26c & 5426795 & 2.3 & 0.24 & 0.0 & 0.0 & tai $15 \mathrm{a}$ & 388214 & 7.28 & 0.89 & 0.02 & 0.00 \\
\hline bur26d & 3821225 & 2.18 & 0.42 & 0.0 & 0.0 & tai $15 b$ & 51765268 & 1.05 & 0.15 & 0.0 & 0.0 \\
\hline bur26e & 5386879 & 2.14 & 0.42 & 0.0 & 0.0 & tai $20 \mathrm{a}$ & 703482 & 12.04 & 0.73 & 0.4 & 0.36 \\
\hline bur26f & 3782044 & 2.39 & 0.59 & 0.0 & 0.0 & tai20b & 122455319 & 9.67 & 1.04 & 0.0 & 0.0 \\
\hline bur $26 \mathrm{~g}$ & 10117172 & 2.07 & 0.22 & 0.0 & 0.0 & tai25a & 1167256 & 11.62 & 0.45 & 0.81 & 0.62 \\
\hline bur $26 \mathrm{~h}$ & 7098658 & 2.27 & 0.4 & 0.0 & 0.0 & tai $25 b$ & 344355646 & 27.12 & 4.34 & 0.0 & 0.0 \\
\hline chr $25 \mathrm{a}$ & 3796 & 168.41 & 11.79 & 0.25 & 0.25 & tai $30 \mathrm{a}$ & 1818146 & 12.25 & 0.41 & 0.89 & 0.35 \\
\hline els19 & 17212548 & 27.04 & 4.91 & 0.0 & 0.0 & tai30b & 637117113 & 25.18 & 3.28 & 0.0 & 0.0 \\
\hline kra30a & 88900 & 26.85 & 1.09 & 0.16 & 0.16 & tai35a & 2422002 & 13.19 & 0.38 & 1.24 & 0.30 \\
\hline kra30b & 91420 & 24.98 & 1.23 & 0.02 & 0.02 & tai35b & 283315445 & 27.86 & 2.42 & 0.0 & 0.0 \\
\hline wil50 & 48816 & 8.69 & 0.26 & 0.04 & 0.04 & tai $40 \mathrm{a}$ & 3139370 & 13.52 & 0.32 & 1.23 & 0.37 \\
\hline nug30 & 6124 & 16.85 & 0.79 & 0.04 & 0.04 & tai40b & 637250948 & 35.94 & 2.3 & 0.0 & 0.0 \\
\hline nug20 & 2570 & 11.65 & 0.95 & 0.0 & 0.0 & tai50a & 4938796 & 13.87 & 0.27 & 1.63 & 0.22 \\
\hline sko42 & 15812 & 16.54 & 0.46 & 0.03 & 0.03 & tai50b & 458821517 & 34.88 & 1.9 & 0.00 & 0.00 \\
\hline sko49 & 23386 & 15.52 & 0.42 & 0.15 & 0.07 & tai $60 \mathrm{a}$ & 7205962 & 13.54 & 0.3 & 1.69 & 0.38 \\
\hline sko56 & 34458 & 15.88 & 0.43 & 0.15 & 0.12 & tai $60 \mathrm{~b}$ & 608215054 & 36.5 & 1.28 & 0.0 & 0.0 \\
\hline sko64 & 48498 & 14.68 & 0.42 & 0.14 & 0.12 & tai $80 \mathrm{a}$ & 13499184 & 12.46 & 0.18 & 1.81 & 0.26 \\
\hline sko72 & 66256 & 14.52 & 0.26 & 0.24 & 0.11 & tai80b & 818415043 & 34.48 & 0.94 & 0.07 & 0.06 \\
\hline sko81 & 90998 & 13.98 & 0.28 & 0.23 & 0.13 & tai $100 \mathrm{a}$ & 21052466 & 11.68 & 0.14 & 1.58 & 0.25 \\
\hline sko90 & 115534 & 13.79 & 0.23 & 0.25 & 0.16 & tai $100 \mathrm{~b}$ & 1185996137 & 33.03 & 0.96 & 0.23 & 0.13 \\
\hline
\end{tabular}

\begin{tabular}{|c|c|c|c|c|c|c|c|c|}
\hline Instance & Bkikv & Best & Average & Warst & SD & PDave(\%) & PDbest(\%) & time(s) \\
\hline bur $20 \mathrm{sa}$ & $542 \times 6570$ & 5426670 & 5426650.00 & 5.4266670 & 0.0 & a.o & 0.0 & 0.55 \\
\hline & 178552 & 3817852 & 3817852.00 & 3817852 & & & & 076 \\
\hline beur $2 \times s e$ & 5426795 & 5426795 & 5426395.00 & 5.426095 & 00 & ao & $\infty$ & 1.16 \\
\hline burrased & 3821225 & $\frac{3821225}{53818}$ & 3821225.00 & 3821225 & 20 & a.o & o. & 177 \\
\hline berr 260 & $\begin{array}{r}53868579 \\
3787094\end{array}$ & 5386879 & 5386879.00 & 5386879 & Q.o & a.o & D. & 1.18 \\
\hline $\begin{array}{l}\text { bur } 206 \mathrm{f} \\
\mathrm{bur} 20 \mathrm{~g}\end{array}$ & $\begin{array}{l}3782044 \\
10117172\end{array}$ & $\begin{array}{l}3782044 \\
10117172\end{array}$ & $\begin{array}{l}37852044,00 \\
10117172.00\end{array}$ & $\begin{array}{l}3782044 \\
10117172\end{array}$ & $\begin{array}{l}20 \\
0.0\end{array}$ & $\begin{array}{l}\text { ao } \\
\text { a.o }\end{array}$ & $\begin{array}{l}\infty .0 \\
0.0\end{array}$ & $\begin{array}{l}0.00 \\
173\end{array}$ \\
\hline 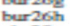 & 709865s & $\begin{array}{l}100117172 \\
7098658\end{array}$ & 70986558.00 & 7098658 & $\begin{array}{l}20 \\
0.0\end{array}$ & 00 & 00 & 075 \\
\hline $\begin{array}{l}\text { chr } 25 a \\
\text { cha }\end{array}$ & 3796 & 3796 & 3805.75 & 3874 & 975 & 025 & 00 & 1376 \\
\hline els 19 & 17212548 & 17212548 & 172125400 & 17212548 & 00 & $\alpha$ & 0.0 & 0.22 \\
\hline kra30a & 88900 & 85900 & 8904875 & 90090 & 148.75 & a.16 & o.a & 9.56 \\
\hline kra30b & 91420 & 91420 & 91446.25 & 91490 & 26.25 & 0.02 & o. & 3005 \\
\hline $\begin{array}{l}\text { wilso } \\
\text { nuse30 }\end{array}$ & 61246 & $\begin{array}{l}458116 \\
6124\end{array}$ & $\begin{array}{l}4883975 \\
612650\end{array}$ & $\begin{array}{l}485866 \\
6128\end{array}$ & $\frac{2375}{25}$ & $\begin{array}{l}0.04 \\
0.04\end{array}$ & oo & $\begin{array}{l}22050 \\
3990\end{array}$ \\
\hline 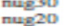 & $\begin{array}{l}6124 \\
2570\end{array}$ & $\begin{array}{l}6124 \\
2576\end{array}$ & $\begin{array}{l}612630 \\
257000\end{array}$ & $\begin{array}{l}6128 \\
2570\end{array}$ & 25 & 004 & a & 3990 \\
\hline tais & 224416 & 224416 & 224416 & $\begin{array}{l}2570 \\
224416\end{array}$ & $\begin{array}{l}0.0 \\
0.0\end{array}$ & $\begin{array}{l}0.8 \\
0.0\end{array}$ & Da & $\begin{array}{l}0.67 \\
0.02\end{array}$ \\
\hline$=12 \mathrm{~b}$ & 39464925 & 39464925 & 39464925 & 39464925 & 00 & a.o & 00 & 003 \\
\hline taisa & 388214 & 358214 & 388224.8 & 385250 & 1297 & 0.02 & o. o & 4.16 \\
\hline tailsb & 51765268 & 517652068 & 51765268 & $\begin{array}{l}517652088 \\
705654\end{array}$ & 20 & ao & o. & 0.06 \\
\hline was & 703482 & 703482 & $\begin{array}{l}706342.0 \\
172458319\end{array}$ & 70sess & 2565.75 & 0.40 & o. & 19.94 \\
\hline 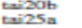 & $\begin{array}{l}122455319 \\
1167256\end{array}$ & $\begin{array}{l}1222455319 \\
1167256\end{array}$ & $\begin{array}{l}1224553319 \\
1176732.8\end{array}$ & $\begin{array}{l}122455319 \\
1181894\end{array}$ & $\frac{200}{7316.99}$ & $\begin{array}{l}0.0 \\
0.81\end{array}$ & $\begin{array}{l}\text { o. } \\
0.0\end{array}$ & $\begin{array}{l}2.44 \\
39.45\end{array}$ \\
\hline tains 25 & 344355646 & 3443556.46 & 344355646 & 344355646 & 00 & 00 & . & 1,3 \\
\hline 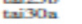 & 1818146 & 1827962 & 1834456.2 & 1843032 & 6474.19 & a.89 & 0.54 & 74.01 \\
\hline tais $30 \mathrm{~b}$ & 637117113 & 637117113 & 637117113 & 637117113 & 00 & ao & 0. & 4.93 \\
\hline $\tan 35 a$ & 24220002 & 24443444 & 24522223.4 & 2460532 & 7270.30 & 1.24 & 0.9 & 95.63 \\
\hline taissb & 283315445 & $2 \times 3315445$ & 283315445 & 283315445 & 20 & a.o & 0.0 & 1052 \\
\hline 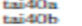 & $\begin{array}{l}3139370 \\
637250948\end{array}$ & $\begin{array}{l}31643722 \\
637250948\end{array}$ & $\begin{array}{l}31782339.4 \\
637250948\end{array}$ & $\begin{array}{l}3192798 \\
637250948\end{array}$ & $\begin{array}{l}11917.91 \\
90\end{array}$ & 1.23 & 0.79 & 140.86 \\
\hline taisola & $\begin{array}{l}637230948 \\
4938796\end{array}$ & 5005070 & $\begin{array}{l}0372503948 \\
5019579.6\end{array}$ & $\begin{array}{l}637250948 \\
5027586\end{array}$ & 1128249 & $\begin{array}{l}0.03 \\
163\end{array}$ & 1.34 & 258518 \\
\hline taisob & 458821517 & 458821517 & 458842516.4 & 458969332 & 20999.39 & 0.00 & 0.0 & 163532 \\
\hline teisoa & 72058 & 3297 & 732 & 735 & 27787.29 & 1.09 & 1.27 & 433.493 \\
\hline taicob & 6082 & $\cos 2$ & 54.0 & 254 & oo & ao & o. o & 133.697 \\
\hline taiste & $\begin{array}{l}1855928 \\
13490184\end{array}$ & 1855928 & 1855928.0 & 1855928 & 2o & 00 & 0.0 & 11506 \\
\hline $580 a$ & 13495 & $\begin{array}{l}13705856 \\
81841=043\end{array}$ & $\begin{array}{l}1374 \\
8189\end{array}$ & $\begin{array}{l}13781508 \\
823187691\end{array}$ & 3505082 & 181 & 1.33 & 1067.45 \\
\hline $\begin{array}{l}\text { tasob } \\
\text { aillooa }\end{array}$ & $\begin{array}{l}818415043 \\
21052466\end{array}$ & $\begin{array}{l}818415043 \\
21322258\end{array}$ & $\begin{array}{l}818994242.5 \\
21385568.0\end{array}$ & $\begin{array}{l}823187691 \\
21421678\end{array}$ & $\begin{array}{l}55164311 \\
53170.40\end{array}$ & $\begin{array}{l}0.07 \\
158\end{array}$ & $\begin{array}{l}0.0 \\
1.28\end{array}$ & $\begin{array}{l}1033.37 \\
1993.95\end{array}$ \\
\hline tail 1000 & 1185996137 & 1187179912 & 1188575434.9 & $\begin{array}{l}1189986795 \\
11898\end{array}$ & 1561365.85 & 0.23 & 0.09 & 2221.83 \\
\hline$=042$ & 15812 & 15812 & 158167 & 15844 & 4.75 & 0.03 & 0.0 & 87.06 \\
\hline $\operatorname{skc} 29$ & 23386 & 23398 & 23421.5 & 23450 & 17.80 & 0.15 & o.as & 245.17 \\
\hline 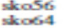 & $\begin{array}{l}34458 \\
48498\end{array}$ & $\begin{array}{l}344660 \\
48502\end{array}$ & $\begin{array}{l}345110 \\
485505\end{array}$ & $\begin{array}{l}3.4584 \\
48686\end{array}$ & $\begin{array}{l}4216 \\
6053\end{array}$ & $\begin{array}{l}0.15 \\
0.14\end{array}$ & $\begin{array}{l}0.02 \\
0.00\end{array}$ & $\begin{array}{l}356.25 \\
521.09\end{array}$ \\
\hline ske & 66256 & 66332 & 604200 & 66490 & 7925 & 0.24 & 0.11 & $820-53$ \\
\hline & 90998 & 91088 & 912 & 91380 & 120.75 & 0.23 & 0.09 & 1090.11 \\
\hline & 34 & 2 & 1158 & 116042 & $18 \times 0$ & 02 & 0.04 & \\
\hline$=0100 a$ & 152002 & 152324 & 152470.2 & 1526992 & 121.95 & 0.30 & 0.21 & 2287.00 \\
\hline
\end{tabular}

\section{Conclusion}

Random Key Cuckoo Search (RKCS) is designed to be easily adapted to solve many combinatorial optimization problems such as the Travelling Salesman Problem and the Quadratic Assignment Problem. Indeed, we have shown that it is easy to move in the continuous space by using Le'vy flights and project these moves in appropriate combinatorial space, of the treated problem, via random key. It proved a good balance between intensification and diversification through Le'vy flights. In this paper, we proposed an application of RKCS to solve QAP. During the design of RKCS, we have focused on the possibility of facilitating the reuse of this metaheuristic in a direct adaptation to solve various combinatorial optimization problems. 
Aziz Ouaarab, Belaid Ahiod, Xin-She Yang, Random Key Cuckoo Search for the Quadratic Assignment Problem.

Transactions on Machine Learning and Artificial Intelligence, Vol 5 No 4 August (2017); p: 842-851

The results are promising, but there is still room for improvement. For example, for the moment, the random key is uniform, and it may be useful to investigate if how any non- uniform decoding of random keys may affect the performance.

In addition, it will be also useful to extend the proposed approach to study larger scale benchmarks. Furthermore, the proposed RKCS may also be useful to solve other combina- torial optimization problems.

\section{REFERENCES}

[1] Ahmed, Z.: A simple genetic algorithm using sequential constructive crossover for the quadratic assignment problem. Journal of Scientific and Industrial Research (2014)

[2] Avriel, M.: Nonlinear programming: analysis and methods. Courier Dover Publications (2012)

[3] Bean, J.: Genetic algorithms and random keys for sequencing and optimization. ORSA journal on computing 6, 154-154 (1994)

[4] Blum, C., Roli, A.: Metaheuristics in combinatorial optimization: Overview and conceptual comparison. ACM Computing Surveys (CSUR) 35(3), 268-308 (2003)

[5] Brixius, N.W., Anstreicher, K.M.: The Steinberg wiring problem. SIAM (2001)

[6] Brown, C.T., Liebovitch, L.S., Glendon, R.: Le'vy flights in dobe ju/?hoansi foraging patterns. Human Ecology 35(1), 129-138 (2007)

[7] Burkard, R.E.: Quadratic assignment problems. Springer (2013)

[8] Burkard, R.E., Karisch, S.E., Rendl, F.: Qap-a quadratic assignment problem library. Journal of Global Optimization 10(4), 391-403 (1997)

[9] Burkard, R.E., Offermann, D.M.J.: Entwurf von schreibmaschinen- tastaturen mittels quadratischer zuordnungsprobleme. Zeitschrift fu" r Operations Research 21(4), B121-B132 (1977)

[10] Christofides, N., Benavent, E.: An exact algorithm for the quadratic assignment problem on a tree. Operations Research 37(5), 760-768 (1989)

[11] Demirel, N.C, ., Toksarı, M.D.: Optimization of the quadratic assignment problem using an ant colony algorithm. Applied Mathematics and Computation 183(1), 427-435 (2006)

[12] Eiselt, H.A., Laporte, G.: A combinatorial optimization problem arising in dartboard design. Journal of the Operational Research Society pp. 113-118 (1991)

[13] Elshafei, A.N.: Hospital layout as a quadratic assignment problem. Operational Research Quarterly pp. 167-179 (1977)

[14] FINKE, G., Burkard, R., Rendl, F.: Quadratic assignment problems. Surveys in combinatorial optimization p. $61(2011)$

[15] Gambardella, L.M., Taillard, E., Dorigo, M.: Ant colonies for the quadratic assignment problem. Journal of the operational research society pp. 167-176 (1999)

[16] Helal, A.M., Abdelbar, A.M.: Incorporating domain-specific heuristics in a particle swarm optimization approach to the quadratic assignment problem. Memetic Computing 6(4), 241-254 (2014)

[17] Hochbaum, D.S.: Approximation algorithms for NP-hard problems. PWS Publishing Co. (1996) 
Transactions on Machine Learning and Artificial Intelligence Vol 5 No 4, Aug 2017

[18] Krarup, J., Pruzan, P.M.: Computer-aided layout design. In: Mathemat- ical programming in use, pp. 75-94. Springer (1978)

[19] Layeb, A.: A novel quantum inspired cuckoo search for knapsack problems. International Journal of BioInspired Computation 3(5), 297-305 (2011)

[20] Li, X., Yin, M.: A hybrid cuckoo search via le'vy flights for the permutation flow shop scheduling problem. International Journal of Production Research 51(16), 4732-4754 (2013)

[21] Mamaghani, A.S., Meybodi, M.R.: Solving the quadratic assignment problem with the modified hybrid pso algorithm. In: Application of Information and Communication Technologies (AICT), 2012 6th International Conference on, pp. 1-6. IEEE (2012)

[22] Maniezzo, V., Colorni, A.: The ant system applied to the quadratic as- signment problem. Knowledge and Data Engineering, IEEE Transactions on 11(5), 769-778 (1999)

[23] Misevicius, A.: Restart-based genetic algorithm for the quadratic assign- ment problem. In: Research and Development in Intelligent Systems XXV, pp. 91-104. Springer (2009)

[24] Nugent, C.E., Vollmann, T.E., Ruml, J.: An experimental comparison of techniques for the assignment of facilities to locations. Operations research 16(1), 150-173 (1968)

[25] Ouaarab, A., Ahiod, B., Yang, X.S.: Discrete cuckoo search algorithm for the travelling salesman problem. Neural Computing and Appli- cations pp. 1-11 (2013). DOI 10.1007/s00521-013-1402-2. URL http://dx.doi.org/10.1007/s00521-013-1402-2

[26] Ouaarab, A., Ahiod, B., Yang, X.S.: Improved and discrete cuckoo search for solving the travelling salesman problem. In: Cuckoo Search and Firefly Algorithm, pp. 63-84. Springer (2014)

[27] Ouaarab, A., Ahiod, B., Yang, X.S.: Random-key cuckoo search for the travelling salesman problem. Soft Computing pp. 1-8 (2014). DOI 10.1007/s00500-014-1322-9. URL http://dx.doi.org/10.1007/s00500014-1322-9

[28] Shlesinger, M.F., Zaslavsky, G.M., Frisch, U.: Le'vy flights and related topics in physics. In: Levy flights and related topics in Physics, vol. 450 (1995)

[29] Skorin-Kapov, J.: Tabu search applied to the quadratic assignment problem. ORSA Journal on computing 2(1), 33-45 (1990)

[30] Taillard, E.: Robust taboo search for the quadratic assignment problem. Parallel computing 17(4), 443-455 (1991)

[31] Taillard, E.: Robust taboo search for the quadratic assignment problem. Parallel computing 17(4), 443-455 (1991)

[32] Taillard, E.D.: Comparison of iterative searches for the quadratic assign- ment problem. Location science 3(2), 87-105 (1995)

[33] Wilhelm, M.R., Ward, T.L.: Solving quadratic assignment problems by simulated annealing. IIE transactions 19(1), 107-119 (1987)

[34] Yang, X.S., Deb, S.: Cuckoo search via le'vy flights. In: Nature \& Biologically Inspired Computing, 2009. NaBIC 2009. World Congress on, pp. 210-214. IEEE (2009) 